Glaucoma

D Vaideanu and S Fraser

\title{
management in pregnancy: a questionnaire survey
}

Keywords: glaucoma; pregnancy; intraocular pressure; guidelines; questionnaire studies

Background The management of glaucoma in a pregnant woman can pose a challenge for the ophthalmologist. The risks and benefits to the mother and foetus must be weighed. When it is decided that treatment is necessary, there is no consensus as to the safest treatment. This study was designed to garner the experiences and views of UK ophthalmologists with regard to this scenario.

Methods All consultant ophthalmologists in the UK were sent a questionnaire. They were asked if they had previous experience treating pregnant women with glaucoma, what management they had used, what management they would currently employ, and, if they were to use medical treatment, what would their first choice agent be. Results A total of 282 out of 605 questionnaires were returned. Of the respondents, $26 \%$ had previously treated pregnant women with glaucoma. Most (71\%) had continued with the therapy that the women were already on. In all, $34 \%$ had observed the situation and had not needed to treat. When asked what they would currently do in this scenario, $31 \%$ were unsure. A total of $40 \%$ would simply continue the prepregnancy treatment. A total of $45 \%$ of respondents, if they were to use medical treatment, would use beta-blockers, compared to $33 \%$ who would use prostaglandins.

Conclusion Although it is thought to be a relatively rare clinical situation, over a quarter of our respondents had first hand experience of the management of glaucoma in pregnancy. Our results indicate some uncertainty about the optimal strategy. It is unlikely that trials will be performed and we suggest guidelines be produced using current knowledge and modified as evidence appears.

Eye (2007) 21, 341-343. doi:10.1038/sj.eye.6702193; published online 25 November 2005

\begin{abstract}
Glaucoma management in pregnancy: a questionnaire survey
\end{abstract}

The glaucomas are a group of diseases characterised by excavation of the optic nerve head and consequent visual field loss. There are a number of risk factors for the disease but the most common is increased intraocular pressure (IOP). Studies have indicated that reduction of IOP can prevent the onset of glaucomatous disc damage ${ }^{1}$ or slow the rate of further damage once it has occurred. ${ }^{2}$

IOP can be lowered in three ways:

1. Medically - either by reducing aqueous fluid production or by increasing its outflow.

2. Surgically - by creating a new outflow channel for the aqueous.

3. Laser-either by reducing aqueous production or by increasing outflow.

All these treatments have risks and benefits that need to be considered in every patient, but are particularly difficult to assess in a pregnant woman who has glaucoma or ocular hypertension. Although this is not a common occurrence, glaucoma affects less than $0.5 \%$ of the population under 40 years of age, ${ }^{3}$ it has the potential to pose a difficult dilemma for the ophthalmologist.

There are, as yet, no evidence-based guidelines on how to treat women in this situation. As a first step in correcting this, we conducted a national survey of current practices and opinions of UK ophthalmologists.

\section{Method}

A postal questionnaire was sent to all consultant ophthalmologists in UK, identified from the
Sunderland Eye Infirmary, Sunderland, Tyne and Wear, UK

Correspondence: S Fraser Sunderland Eye Infirmary, Queen Alexandra Road, Sunderland, Tyne and Wear SR2 9HP, UK Tel: + 44191569 9853; Fax: +44 1915699060 . E-mail: sfraser100@ totalise.co.uk

Received: 20 June 2005 Accepted in revised form: 18 October 2005 Published online: 25 November 2005 
Medical Directory and internet database search (www.specialistinfo.com). They were asked:

1. Have you ever managed a pregnant woman with glaucoma or ocular hypertension?

2. What was your management?

3. If you were faced with a scenario in which a pregnant woman had a level of IOP that was felt likely to cause disease progression, which management strategies would you consider initially?

4. If one of the answers to the above was 'medical treatment', they were asked which medication would be their first choice

The study had ethical committee approval.

\section{Results}

A total of 605 questionnaires were sent out and 306 were returned. Of these, 282 (47\%) were completed correctly. There was an equal spread of replies across the ophthalmology units in the UK.

Of the 282 who replied correctly, 73 (26\%) consultant ophthalmologists had previously treated pregnant women with glaucoma. Of these (note: some ophthalmologists treated more than one patient):

- 25 (34\%) had simply observed the patient(s) that is, no medical or surgical intervention.

- $52(71 \%)$ had continued with medical treatment(s).

- Nine $(12 \%)$ had used surgery or laser

- Two (3\%) could not recall what treatment they had used.

When questioned about the scenario in which a pregnant woman had a level of IOP which was felt likely to cause disease progression during her pregnancy, the management strategies that respondents would consider were as follows:

- $17 \%$ stated they would still initially observe the patient.

- $40 \%$ would use (or continue to use) topical treatment.

- $8 \%$ would use laser trabeculoplasty (LTP).

- $4 \%$ would choose surgery.

- $31 \%$ of the doctors were unsure of what treatment option they would choose.

Of the 25 who would be prepared to use medical treatment,

- $45 \%$ would use topical beta-blockers first.

- $33 \%$ would use topical prostaglandin's first.

- $22 \%$ would use other medications first.

\section{Discussion}

The essential problem in treating pregnant women with glaucoma is balancing the risks of treatment to the mother and baby with the danger of the glaucoma progressing during the pregnancy. Our results indicate that this is not such an uncommon dilemma, with $26 \%$ of our respondents previously encountering this scenario.

All glaucoma medications present potential hazards to the foetus, ${ }^{4-7}$ as do local or general anaesthetics.

Therefore, ideally it is best to avoid any interventions in pregnant women. Despite the fact that the woman may already be on treatment for high IOP, this strategy may still be possible. A number of studies have shown that there is often a spontaneous decrease in IOP during pregnancy. ${ }^{8-10}$ This is believed to be because of a combination of increased uveoscleral outflow (postulated to be a result of hormonal changes), decreased episcleral pressure secondary to general decrease of venous pressure in upper extremities and the acidosis of pregnancy. ${ }^{8-10}$ This is consistent with the fact that $34 \%$ of our respondents had previously not needed to intervene during the pregnancy and $17 \%$ would currently use this as a first-line strategy - even if glaucomatous damage was already present.

Unfortunately, in a proportion of woman, glaucomatous damage may be so severe (or the IOP so high) that the risk of further visual field progression during the pregnancy is too high. In this situation, the IOP needs to be lowered, and our results show (as does the literature) that there is some uncertainty as to the optimum method to achieve this.

Our survey indicated that $71 \%$ of the respondents, who had previously treated pregnant women, had continued their medical therapy during the pregnancies (most pregnant women with glaucoma are already diagnosed, and development of the disease in pregnancy is very rare $^{7}$ ). A total of $40 \%$ would use this as their initial strategy in the future. The majority of medical treatments to reduce IOP have been shown to be potentially hazardous to the foetus. ${ }^{6}$

When asked which topical antihypertensive they were happiest to use in pregnant women, $45 \%$ named topical beta-blockers. This is despite the fact that ophthalmologists are increasingly using topical prostaglandin agonists as their first-line drug for lowering IOP. ${ }^{11}$ It is likely that there is a perception that beta-blockers are safer in pregnant woman than prostaglandins - perhaps because of the well-known use of prostaglandins in the termination of pregnancy.

Despite this latter point, 25\% would be willing to use topical prostaglandins in pregnant women.

Some respondents $(12 \%)$ had previously used laser or surgery, and $8 \%$ would be happy to use this again if necessary. Surgical treatment requires local or general anaesthetic, both of which pose hazards to the mother and foetus. ${ }^{7}$ LTP has been considered as an alternative to medical therapy but the effects may be temporary and 
other medications required. ${ }^{7}$ Cyclodiode laser has also been used. ${ }^{12}$

There are a number of potential biases in this study. Postal questionnaires often have a low response rate; our response rate of nearly $50 \%$ is reasonably good for surveys such as this. ${ }^{13}$ It may be surmised that nonrespondents were less likely to have encountered this scenario (so they did not reply), and it may therefore be that this condition is encountered less often than our results suggest (26\%). The results should also be interpreted in the light of the possible recall bias of the respondents - it may have been some time since they had treated the patient(s).

Perhaps the most notable result of our survey is the uncertainty of ophthalmologists faced with a pregnant woman who has glaucoma-34\% reported being unsure of how they would manage the situation. To date, there are no guidelines for the management of glaucoma in pregnant women; this is presumably because they represent a small number of patients in whom it is difficult to do clinical trials. It is often in this scenario that consensus guidelines are most useful to the practitioner. However, as a first step we would suggest that there should be an attempt to gauge how often this situation occurs nationally and to attempt to monitor any adverse outcomes (from treatment or lack of treatment). This would perhaps be best carried out by a system that can pick up relatively rare events, such as the British Ophthalmic Surveillance Unit (BOSU).

In conclusion, although glaucoma is relatively uncommon in women of child-bearing age, one in four of our respondents had had to deal with it in pregnant women. As always in pregnancy, treatment can be challenging, with the risks of damage to the mother and/ or foetus needing to be balanced with the risks of disease progression during the pregnancy. It may be possible to stop treatment during pregnancy because of the physiological drop in IOP. If this is not enough, all IOPlowering options have potential hazards. Our survey indicates that there is a general level of uncertainty among ophthalmologist about this situation. We suggest that in the short term, some attempt be made to produce consensus guidelines that can be modified in the light of future research into this challenging situation.

\section{References}

1 Gordon MO, Beiser JA, Brandt JD, Heuer DK, Higginbotham EJ, Johnson CA et al. The Ocular Hypertension Treatment Study: baseline factors that predict the onset of primary open-angle glaucoma. Arch Ophthalmol 2002; 120(6): 714-720.

2 Nouri-Mahdavi K, Hoffman D, Coleman AL, Liu G, Li G, Gaasterland D et al. Predictive factors for glaucomatous visual field progression in the Advanced Glaucoma Intervention Study. Ophthalmology 2004; 111(9): 1627-1635.

3 Werner DL, Sherman J. Prevalence of glaucoma in optometric patients under 40 years of age. Am J Optom Physiol Opt 1977; 54: 767-770.

4 Wagenvoort AM, Van Vugt JM, Sobotka M, van Geijn HP. Topical timolol therapy in pregnancy: is it safe for the fetus? Teratology 1998; 58(6): 258-262.

5 De Santis M, Lucchese A, Carducci B, Cavaliere AF, De Santis L, Merola A et al. Latanoprost exposure in pregnancy. Am J Ophthalmol 2004; 138(2): 305-306.

6 Chung CY, Kwok AK, Chung KL. Use of ophthalmic medications during pregnancy. Hong Kong Med J 2004; 10(3): 191-195.

7 Johnson SM, Martinez M, Freedman S. Management of glaucoma in pregnancy and lactation. Surv Ophthalmol 2001; 45(5): 449-454.

8 Becker B, Friedenweld JS. Clinical aqueous outflow. Arch Ophthalmol 1953; 50: 557-571.

9 Green K, Philips CI, Cheeks L, Slage T. Aqueous humour flow rate and intraocular pressure during and after pregnancy. Ophthalmic Res 1988; 20: 353-357.

10 Sunness JS. The pregnant women's eye. Surv Ophthalmol 1988; 32(4): 219-238.

11 Kaiserman I, Kaiserman N, Nakar S, Vinker S. The effect of combination pharmacotherapy on the prescription trends of glaucoma medications. J Glaucoma 2005; 14(2): 157-160.

12 Wertheim M, Broadway D. Cyclodiode laser therapy to control intraocular pressure during pregnancy. $\mathrm{Br} \mathrm{J}$ Ophthalmol 2002; 86(11): 1318-1319.

13 McAvoy BR, Kaner EF. General practice postal surveys: a questionnaire too far? BMJ 1996; 313: 732-733. 